\title{
IDADES U-Th-Pb E U-Pb EM MONAZITA DE ORTOGNAISSE DO COMPLEXO JAMARI, ÁREA DO DEPÓSITO DE ESTANHO DE BOM FUTURO (RO)
}

\author{
VALMIR DA SILVA SOUZA ${ }^{1}$, LUCIANA MIYAHARA TEIXEIRA ${ }^{2}$, ELTON LUIZ DANTAS \\ NILSON FRANCISQUINI BOTELHO ${ }^{3}$ \& JORGE HENRIQUE LAUX ${ }^{3}$
}

\begin{abstract}
Resumo O método geocronológico U-Th-Pb em monazita, por meio de microssonda eletrônica, vem sendo aplicado no estudo geotermobarométrico de terrenos metamórficos e fornecendo resultados compatíveis com os métodos absolutos de datação geocronológica. Esse trabalho compara os resultados analíticos obtidos por este método com os de U-Pb por diluição isotópica em cristais de monazita de ortognaisse do Complexo Jamari, Paleoproterozóico da região central de Rondônia. Os cristais analisados variam de 0,5 a 1,5 mm, são limpos, não zonados, caracterizando uma Ce-monazita monogenética. A aplicação do método U-Th$\mathrm{Pb}$ revelou idade $\mathrm{Pb}-\mathrm{Th} *$ de $1327 \pm 20 \mathrm{Ma}$, enquanto que pelo método U-Pb obteve-se idade $1331 \pm 0,98 \mathrm{Ma}$. Os valores de idades próximos revelam a eficácia do método U-Th- $\mathrm{Pb}$, apesar da menor precisão, em relação aos métodos de datação absoluta. As idades obtidas são interpretadas como registros de cristalização metamórfica, associada ao evento regional que afetou as rochas do embasamento na região central de Rondônia, durante a orogenia Rondoniana-San Ignácio.
\end{abstract}

Palavras-Chave. Complexo Jamari, Rondônia, depósito de estanho do Bom Futuro, idade U-Th-Pb em monazita, idade U-Pb em monazita

\begin{abstract}
U-Th-Pb AND U-Pb AGES IN MONAZITE FROM ORTHOGNEISS OF THE JAMARI COMPLEX IN THE BOM FUTURO TIN DEPOSIT (RO) Electron microprobe U-Th-Pb dating of monazite is applied to the geothermobarometric study of metamorphic terrains and provides important results, compatible with absolute geochronological methods. This paper compares the analytic results obtained by eletron microprobe U-Th- $\mathrm{Pb}$ with isotopic dilution $\mathrm{U}-\mathrm{Pb}$ in monazite from orthogneiss of the Paleoproterozoic Jamari Complex, in Rondônia State, Brazil. The monazite grains are rounded, clean and not zoned, ranging from 0,5 to $1,5 \mathrm{~mm}$, characterizing a monogenetic Ce-monazite. The $\mathrm{Pb}-\mathrm{Th}^{*}$ cationic diagram analysis revealed an age of 1327 $\pm 20 \mathrm{Ma}$ and the U-Pb concordia diagram yielded an age of $1331 \pm 0,98 \mathrm{Ma}$. These values, in spite of the lower precision of the method, reinforce the applicability of the U-Th-Pb eletron microprobe analysis in geochronological investigations. The obtained ages are interpreted as records of a tectonometamorphic event that affected the basement rocks in central Rondônia during the Rondonian-San Ignácio orogeny.
\end{abstract}

Keywords. Jamari Complex, Rondônia, Bom Futuro tin deposit, U-Th-Pb age of mozanite, U-Pb age of monazite.

INTRODUÇÃO. A datação por U-Th-Pb em monazita com microssonda eletrônica foi desenvolvida na década de 1990 e despertou interesse, principalmente devido ao modo simples e de baixo custo para obtenção dos dados analíticos, além de permitir análises pontuais em cristais monogenéticos e poligenéticos (Suzuki et al. 1991, Suzuki \& Adachi 1991, Montel et al. 1996, Braun et al. 1998). O método apresenta limitações quanto à incapacidade de distinguir diferentes isótopos $(\mathrm{Pb}$ inicial e $\mathrm{Pb}$ radiogênico) e à baixa precisão analítica (Catlos et al. 2002, Cocherie \& Albarede 2001). O método clássico de obtenção de concórdia $\mathrm{U}-\mathrm{Pb}$ por diluição isotópica em monazita, apesar do custo e do tempo mais elevados, apresenta maior precisão e é amplamente empregado em estudos geocronológicos (Faure 1986). Contudo, no caso de monazita poligenética, o método U-Pb não permite a datação do(s) evento(s) geológico(s) responsável(is) pelo crescimento e/ou recristalização da monazita (Foster et al. 2002).

$\mathrm{O}$ método U-Th- $\mathrm{Pb}$ em monazita tem sido aplicado, principalmente, nas investigações geotermobarométricas de terrenos metamórficos (Braun et al. 1998, Vlach \& Gualda 2000, Tickyj et al. 2001, Vlach \& Del Lama 2002, Foster et al. 2002), fornecendo resultados consistentes com valores obtidos por métodos mais precisos, tais como U-Pb em zircão por SHIRIMP (Vlach \& Correia 2001). Esse trabalho apresenta os dados U-Th- $\mathrm{Pb}$ e $\mathrm{U}-\mathrm{Pb}$ em cristais de monazita de ortognaisse do Complexo Ja- mari, na área do depósito de estanho do Bom Futuro, discutindo os valores obtidos e sua inserção no quadro geocronológico na região central de Rondônia.

PROCEDIMENTO ANALÍTICO. As análises de U-Th$\mathrm{Pb}$ foram realizadas no Laboratório de Microssonda Eletrônica da Universidade de Brasília, em um equipamento CAMECA SX50, dotado de sistema WDS, com ângulo de take off de $40^{\circ}$, voltagem de aceleração de $20 \mathrm{Kv}$, corrente de $100 \mathrm{nA}$ e tempos de contagem de 240s para $\mathrm{Pb}, 200 \mathrm{~s}$ para $\mathrm{U}$ e $40 \mathrm{~s}$ para os demais elementos (Cocherie et al. 1998).

Para o cálculo da idade de cristalização da monazita, utilizou-se o método de Suzuki \& Adachi $(1991,1994)$, no qual as concentrações de $\mathrm{UO}_{2}, \mathrm{ThO}_{2}$ e $\mathrm{PbO}$, obtidas por microssonda eletrônica, são aplicadas para obtenção de uma idade aparente $(\mathrm{t})$, por meio da equação $\mathrm{PbO} / \mathrm{W}_{\mathrm{Pb}}=\mathrm{ThO}_{2} / \mathrm{W}_{\mathrm{rh}}\left(\mathrm{e}_{-}^{232 t}-1\right)+\mathrm{UO}_{2} /$ $\left.\mathrm{W}_{\mathrm{U}}\left(\mathrm{e}^{-235 \mathrm{t}}\right)+138 \mathrm{e}^{238 \mathrm{t}}-1 / 139\right)$, onde $\mathrm{W}^{2}$ é o peso melecular de cada óxido $\left(\mathrm{W}_{\mathrm{Pb}}=224 ; \mathrm{W}_{\mathrm{Th}}=264 \mathrm{e} \mathrm{W}_{\mathrm{U}}=270\right)$, é a constante de decaimento radioativo de cada isótopo $C_{232}=4,9475 \times 10^{-11} /$ ano, ${ }_{-235}=9,8485 \times 10^{-10} /$ ano e ${ }_{-238}=1,55125 \times 10^{-10} / \mathrm{ano}$ ). Conhecendo-se a idade de cada ponto, converte-se o conteúdo de $\mathrm{U}$ em um conteúdo equivalente de Th, o qual produziria a mesma quantidade de $\mathrm{Pb}$ que o $\mathrm{U}$ presente no mineral. Esse Th equivalente é adicionado ao Th medido, fornecendo o Th aparente $\left(\mathrm{Th}^{*}\right)$. O cálculo do $\mathrm{Th}^{*}$ é expresso por meio da equação $\mathrm{ThO}_{2}{ }^{*}$

1 - Universidade Federal do Amazonas, Departamento de Geociências, Av. Gal. Rodrigo O. J. Ramos, 3000, Mini-Campus,CEP:69.077-000, Manaus (AM), e-mail: valmirsouza(a)ufam.edu.br

2 - Instituto Brasileiro de Meio-Ambiente (IBAMA), Av. L4 Norte, Ed. Sede do Ibama, Brasília (DF), e-mail: luciana.miyahara@celaf.ibama.gov.br

3 - Universidade de Brasília, Instituto de Geociências, Campus Universitário Darcy Ribeiro, CEP: 70910-900, Brasília(DF), e-mail: nilsonfb@unb.br, elton@)unb.

bre lauxjh(a) unb.br 

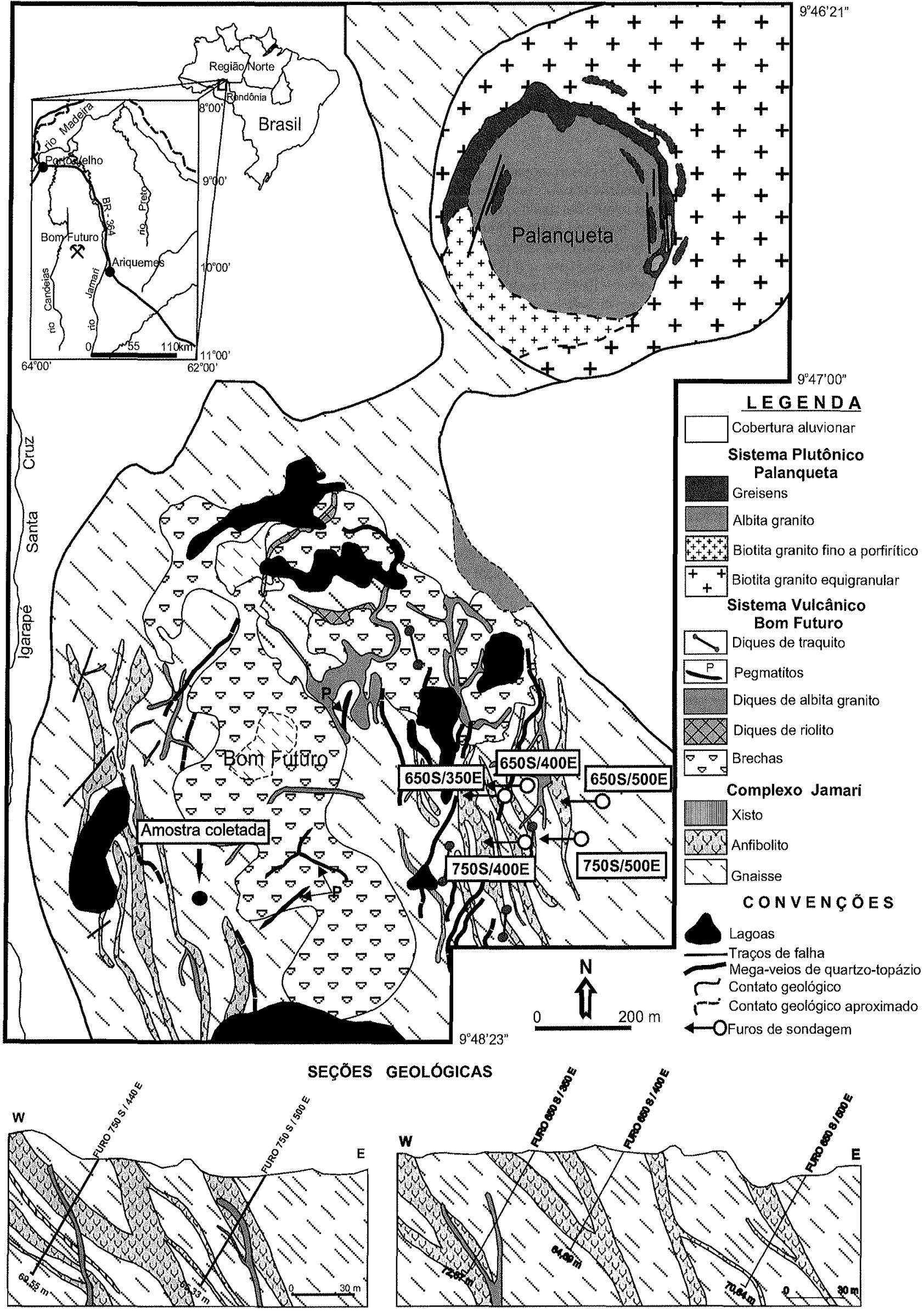

Figura 1. Mapa geológico da área do depósito de estanho de Bom Futuro. 
$=\mathrm{ThO}_{2}+\left\{\left(\mathrm{UO}_{2} \times \mathrm{W}_{\mathrm{Th}}\right) /\left[\mathrm{W}_{\mathrm{U}}\left(\mathrm{e}^{232 t}-1\right)\right]\right\} \times\left\{\left[\left(\mathrm{e}^{235 t}+138 \mathrm{e}^{238 \mathrm{t}}\right) /\right.\right.$ 139] -1 $\}$. Em um diagrama $\mathrm{PbO} \times \mathrm{ThO}_{2}{ }^{*}$, as amostras de mesma idade definem uma reta cuja inclinação está relacionada à idade da monazita. Para o cálculo da linha de regressão, sua inclinação, o grau de incerteza dos valores de (t) e a idade da amostra utilizou-se o programa ISOPLOT (Ludwig 2001).

As análises para obtenção de diagrama concórdia U-Pb foram realizadas no Laboratório de Geocronologia da Universidade de Brasília, utilizando-se cristais individuais de monazita dissolvidos em $\mathrm{H}_{2} \mathrm{SO}_{4}$ concentrado $(5 \mathrm{uL}$ ) ultrapuro e em béquer de Teflon. Foi adicionado um traçador (spike) ${ }^{205} \mathrm{~Pb}^{235} \mathrm{U}$. A solução perma-neceu durante três dias em chapa aquecedora (ca. $125-140^{\circ} \mathrm{C}$ ). Para separação química foi utilizada resina de troca iônica convencional, usando microcolunas de Teflon, seguindo procedimento de Krogh (1973). O Pb and $U$ foram coletados e depositados juntos. Os filamentos utilizados são de Re e o depósito foi feito com $\mathrm{H}_{3} \mathrm{PO}_{4}$ e Si gel. Para leitura foi utilizado um espectrômetro de massa Finnigan MAT-262 com multi-coletor. O branco laboratorial é melhor que $15 \mathrm{pg}$. Os programas PBDAT (Ludwig 1993) e ISOPLOT-Ex (Ludwig 2001) foram usados para o cálculo das idades, cujos erros das razões isotópicas são $2 \sigma$.

CONTEXTO GEOLóGICO. O depósito de estanho do Bom Futuro está localizado no centro-oeste de Rondônia, no município de Ariquemes, cerca de $200 \mathrm{~km}$ a sul de Porto Velho
(Figura 1). É formado por um sistema vulcano-plutônico, encaixado em rochas do Complexo Jamari e circundado por coberturas sedimentares aluviais (Villanova \& Franke 1995, Silva et al. 1995 e 1997, Souza e Botelho 2002, Souza 2003).

O Complexo Jamari foi definido por Isotta et al. (1978), na região central de Rondônia, como embasamento polimetamórfico paleoproterozóico, formado principalmente por rochas ortoderivadas, tais como gnaisses, migmatitos, granulitos e anfibolitos. Entretanto, reconhecimentos geológicos posteriores demonstram que, nesta região, o Complexo Jamari é formado por uma associação de rochas orto e paraderivadas, tais como gnaisses graníticos a granodioríticos, biotita-granada gnaisse, sillimanita-granada gnaisse, granada-cordierita gnaisse, biotititos, kinzigitos, rochas calcissilicáticas, orto-anfibolitos, paramanfibolitos, granada-biotita xistos e granulitos (Scandolara et al. 1996 e 2001, Amorim et al. 1999a e 1999b, Silva et al. 2002, Souza et al. 2005). Estas rochas seriam produtos dos sucessivos episódios de magmatismo, metamorfismo e deformação, ocorridos entre 1,75 e 0,97 Ga, com sedimentação entre 1,67 e 1,57 Ga. (Payolla et al. 2002).

No âmbito do depósito Bom Futuro, o Complexo Jamari é formado pela associação de paragnaisse, xisto, para-anfibolito, ortognaisse e orto-anfibolito, dispostos como corpos lenticulares centimétricos a decamétricos, orientados segundo a direção geral NNW-SSE e mergulhando cerca de $50^{\circ}$ para ENE (Figura 1). Essas rochas estão polideformadas, variando de milonitos
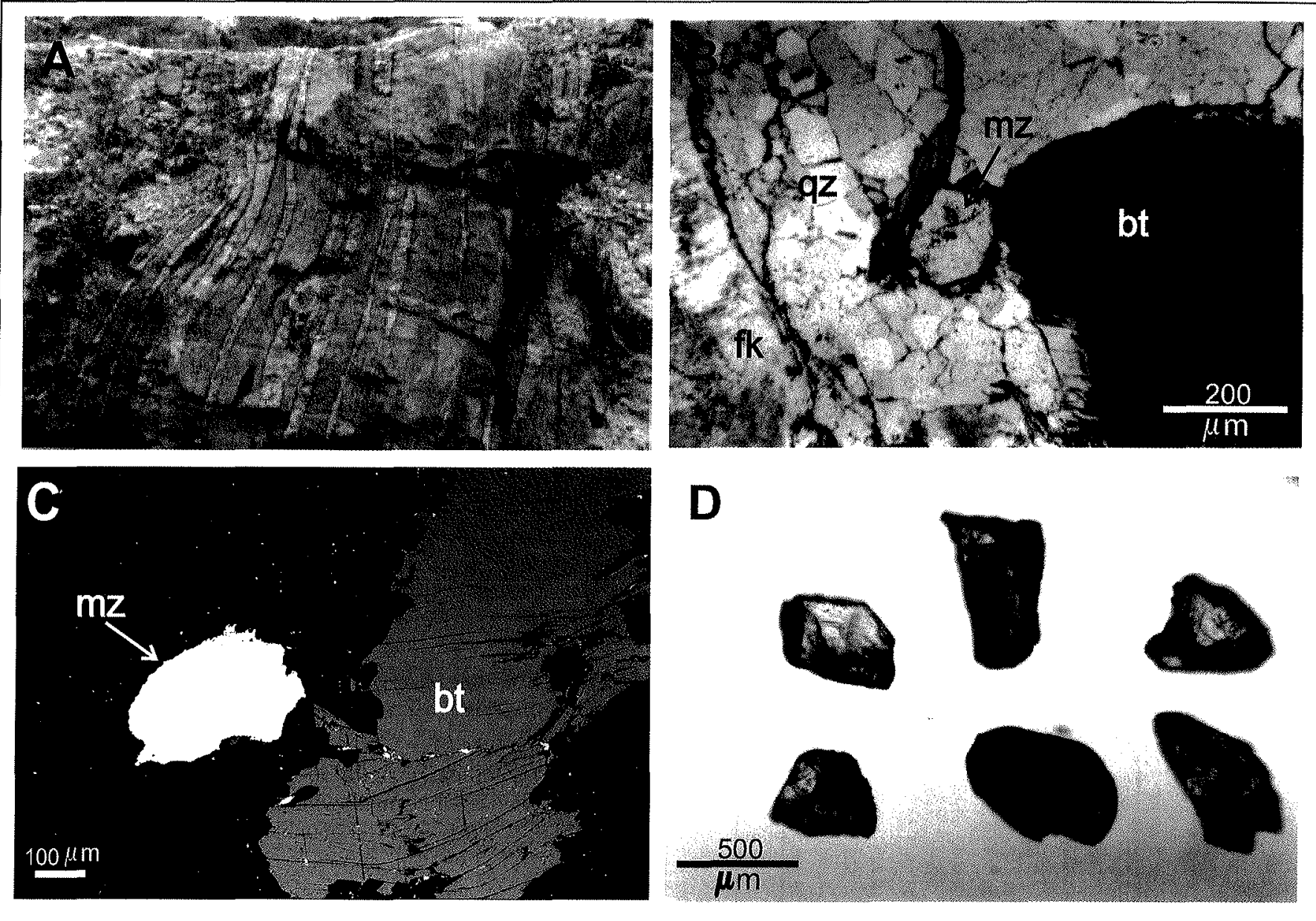

Figura 2. A: ortognaisse do Complexo Jamari na área do depósito de estanho de Bom Futuro; B: fotomicrografia de cristal de monazita (polarizadores paralelos); $C$ : imagem de elétrons retroespalhados (BSE) de cristal de monazita utilizado para as análises por microssonda eletrônica; D: cristais de monazita utilizados na análise isotópica U-Pb. pl = plagioclásio, qz = quartzo, $b t=$ biotita, $f k=$ feldspato potássico, $m z=$ monazita . 


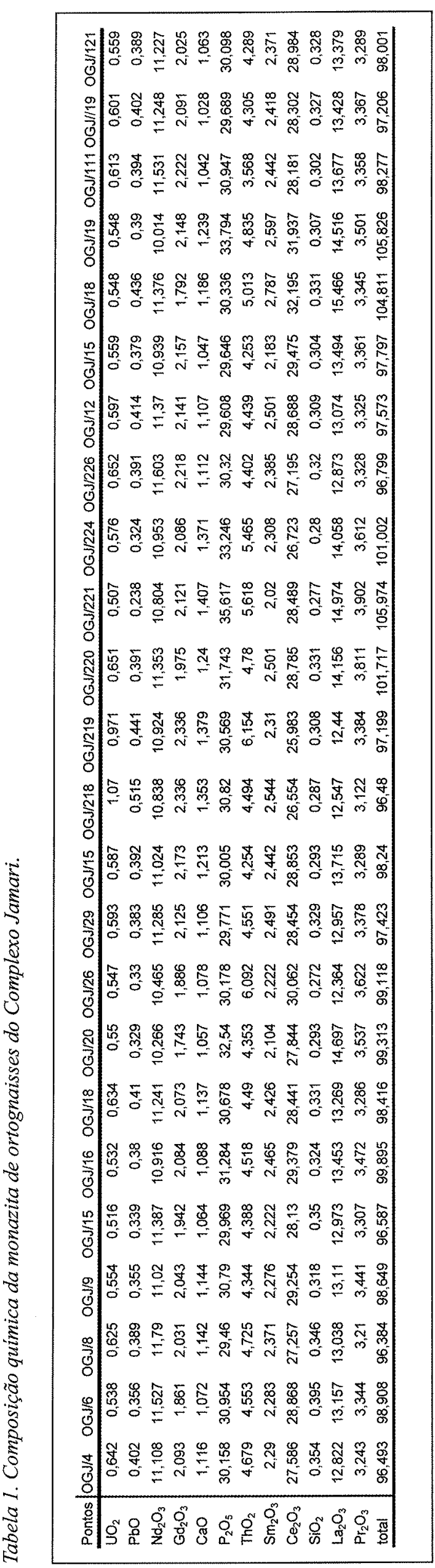

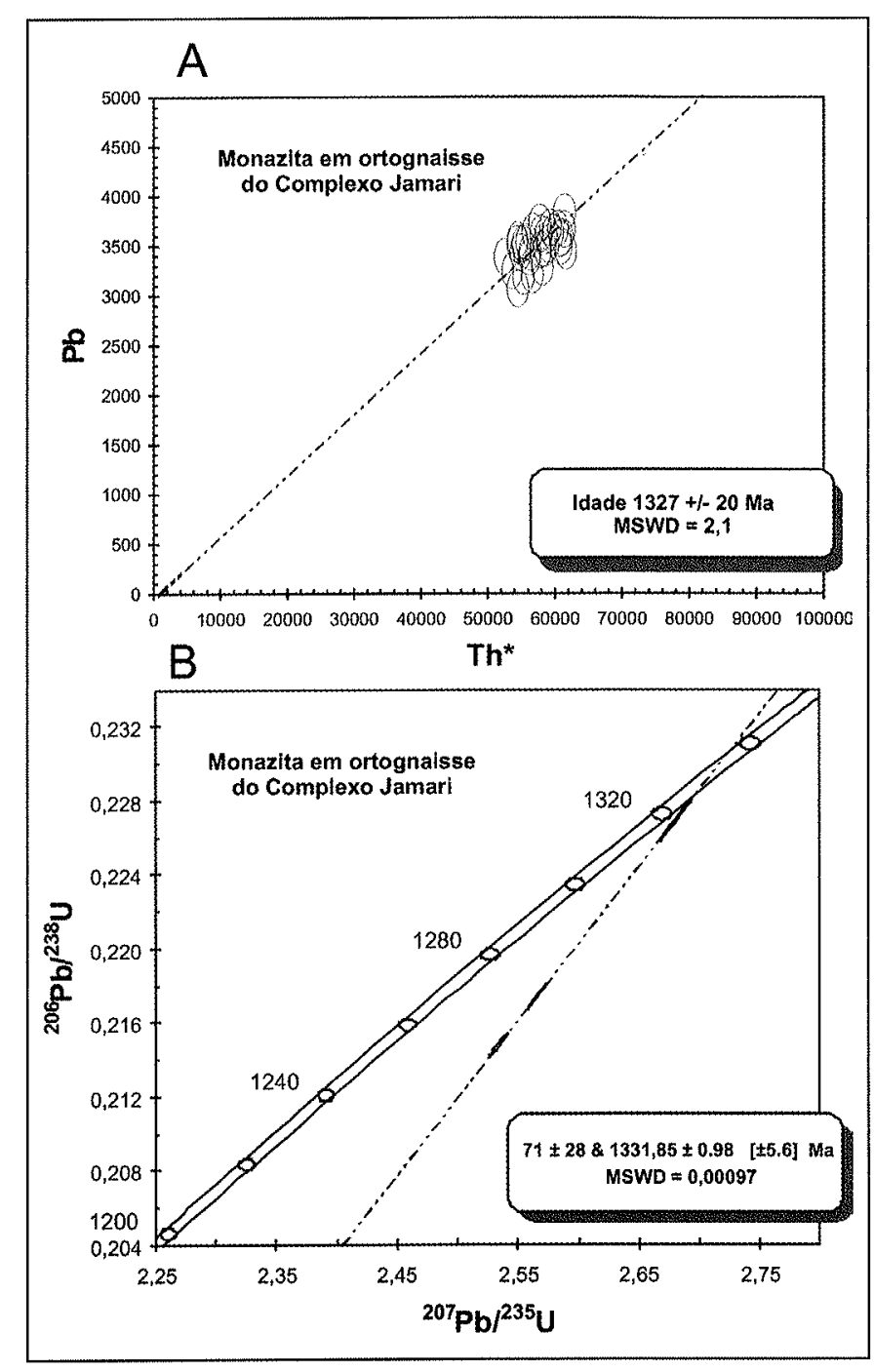

Figura 3. Idades da monazita de ortognaisse do Complexo Jamari. A: diagrama de correlação $\mathrm{Pb}$-Th*, aplicado conforme a método proposto por Suzuki \& Adachi (1991 e 1994); B: diagrama concórdia $\mathrm{U}-\mathrm{Pb}$.

a ultramilonitos, metamorfisadas em médio grau, zona de transição entre as fácies xisto-verde alto e anfibolito baixo. Provavelmente representam um episódio vulcano-sedimentar ainda pouco estudado, com fonte dominantemente paleoproterozóica, idade modelo $\left(\mathrm{T}_{\mathrm{DM}}\right)$ da ordem de 2,0 Ga. e cujos valores de $\mathrm{Nd}(\mathrm{t})$ sugerem derivação de uma crosta continental juvenil (Souza 2003, Souza et al. 2005).

ORTOGNAISSE DO COMPLEXO JAMARI. Gnaisses representam os litotipos predominantes do Complexo Jamari na área do depósito Bom Futuro. Os ortognaisses afloram, principalmente, nas áreas noroeste e centro-norte do sistema vulcânico Bom Futuro (Figura 1), são cinza rosado e possuem marcante bandamento metamórfico, definido pela alternância de faixas quartzo-feldspáticas de cor rosa a vermelho e níveis micáceos (Figura 2A). O bandamento é descontínuo e irregular, milimérico a centimétrico, de granulação fina a média, localmente média a grossa, e define uma foliação $\mathrm{S}_{1}$, entre $\mathrm{N} 15^{\circ} \mathrm{W} / 70^{\circ} \mathrm{SW}$ e $\mathrm{N} 20^{\circ} \mathrm{E} / 50^{\circ} \mathrm{SE}$. Ao microscópio, os ortognaisses apresentam textura heterogranular granolepidoblástica a granoblástica (Figura 2B). São monzograníticos, com ligeira tendência a sienograníticos, constituídos por microclínio pertítico $(35-40 \%)$, quartzo 
(25-30\%), albita-oligoclásio $\left(\mathrm{An}_{08-14} 20-25 \%\right)$ e biotita (5-10 $\%$, tendo como minerais acessórios fengita, zircão, titanita, monazita, apatita, barita, epidoto, fluorita e minerais opacos (pirita, calcopirita, ilmenita).

A amostra utilizada nesse trabalho foi coletada na área centro-norte do sistema vulcânico Bom Futuro, sob as coordenadas geográficas $9^{\circ} 47^{\prime} 44^{\prime \prime} \mathrm{S}$ e $63^{\circ} 33^{\prime} 41^{\prime \prime} \mathrm{W}$ (Figura 1) e representa uma fácies de granulação média a grossa do ortognaisse do Complexo Jamari.

PETROGRAFIA E GEOQUÍMICA DA MONAZITA. A monazita ocorre, normalmente, associada aos cristais de quartzo, biotita e microclínio pertítico, distribuída de forma intersticial e/ ou inclusa em biotita, onde desenvolve discretos halos pleocróicos. Os cristais são subédricos a euédricos, com tamanho entre 0,5 a $1,5 \mathrm{~mm}$, são limpos, não zonados e pouco fraturados (Figura 2B a 2D).

As análises por microssonda eletrônica revelam que a monazita do ortognaisse é rica em elementos terras raras leves, compondo cerca de $70 \%$ de sua composição química (Tabela 1 ), cujas concentrações estão distribuídas em Ce $(25,9$ a $39,2 \%)$, La (11 a $16,8 \%), \mathrm{Nd}(10,3$ a 12,6\%), $\operatorname{Pr}(2,7$ a $4,3 \%), \operatorname{Sm}(2$ a $3,2 \%)$ e Gd (1,7 a 3\%), caracterizando uma Ce-monazita (Figura 2E). Apresentam ainda conteúdos de $\mathrm{CaO}\left(0,8\right.$ a 1,3\%), $\mathrm{ThO}_{2}(3,5 \mathrm{a}$ $6,2 \%), \mathrm{SiO}_{2}(0,3$ a $0,5 \%)$ e $\mathrm{UO}_{2}(0,5$ a $1 \%)$.

GEOCRONOLOGIA U-Th-Pb e U-Pb. Para o cálculo da idade, com base nas concentrações de $\mathrm{UO}_{2}, \mathrm{ThO}_{2}$ e $\mathrm{PbO}$, foram utilizadas apenas as análises com totais acima de $96 \%$ e abaixo de $105 \%$, o que compreende um total de 22 pontos analisados em três cristais de monazita (Tabela 1). O diagrama de correlação entre $\mathrm{Pb}$ e Th* forneceu idade de $1327 \pm 20 \mathrm{Ma}$ (Figura 3A). Neste diagrama, segundo Suzuki \& Adachi (1991 e 1994), o valor de Th* é definido pelo somatório do conteúdo de $\mathrm{ThO}_{2}$ analisado pela microssonda eletrônica e o conteúdo em Th que produziria a mesma quantidade de $\mathrm{Pb}$ que o $\mathrm{UO}_{2}$ presente na amostra.

Para o cálculo da idade isocrônica U-Pb (Tabela 2), utilizando o método clássico de diluição isotópica, foram consumidos cerca de seis cristais de monazita com tamanho em torno de $0,5 \mathrm{~mm}$ (Figura $2 \mathrm{~F}$ ), cujos resultados permitiram a construção do diagrama concórdia U-Pb com idade de $1331 \pm 0,98 \mathrm{Ma}$ (Figura 3B).

CONCLUSÃO. O método de U-Th-Pb em monazita aplicado ao estudo geocronológico forneceu, de modo mais rápido e a custo mais baixo, uma razoável aproximação aos resultados obtidos por métodos absolutos de datação isotópica. Outra vantagem reside no caso de cristais de monazita poligenéticos, onde uma análise prévia por microssonda eletrônica permitirá, por exemplo, detectar variações de idades, auxiliando na identificação de eventos geológicos envolvidos na cristalização e/ou recristalização do mineral (Suzuki \& Adachi 1991, Cocherie \& Albarede 2001, Foster et al. 2002). Os resultados obtidos por microssonda eletrônica $\left(\mathrm{Pb}-\mathrm{Th}^{*}\right.$ de $\left.1327 \pm 20 \mathrm{Ma}\right)$ corroboram com razoável aproximação os de diluição isotópica (U-Pb de $1331 \pm 0,98 \mathrm{Ma})$ em cristais monogenéticos. Estes valores estão também de acordo com as idades U-Pb de $1326 \pm 1 \mathrm{Ma}$ em monazita de ortognaisse (Payolla et al. 2002) e U-Pb SHRIMP de $1321 \pm 27 \mathrm{Ma}$ e $1.332 \pm 11 \mathrm{Ma}$ em zircão de ortognaisse (Silva et al. 2002), obtidas na região centro-norte de Rondônia, interpretadas como registros de cristalização metamórfica, associada ao evento regional que afetou as rochas do embasamento durante a orogenia Rondoniana-San Ignácio (Tassinari et al. 2000, Payolla et al. 2002).

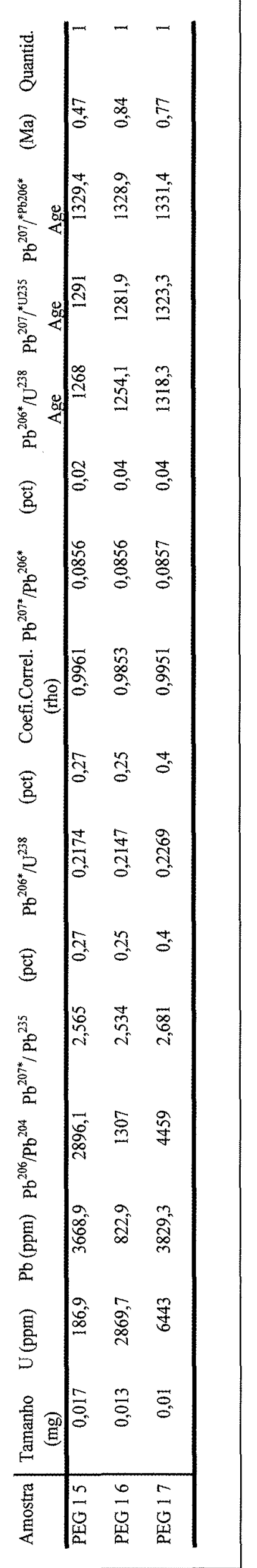


Agradecimentos. Os autores agradecem à Empresa Brasileira de Estanho S.A. (EBESA/Grupo Paranapanema), através do Eng. de Minas Pierre Mathias e do Geol. Renato Muzzolon, pelo total apoio durante a etapa de campo. À Companhia de Pesquisa e Recursos Minerais (CPRM-RO), através dos Geol. Rommel da Silva Sousa, Marcos Quadros, Jânio Leite de Amorim (in memorian), Jaime Scandolara e Antônio Vieira, pelas discussões durante as atividades de campo. Ao $\mathrm{CNPq}$, pelo auxílio financeiro à pesquisa, processos 47519/04 e 303703/2004-0.

\section{Referências}

Amorim J.L., Rizzoto G.J., Scandolara J.E. 1999a. Complexo Jamari, sudeste do Cráton Amazônico: reavaliação do contexto geotectônico.In: SBG (BA), Simp. Nac. Estudos Tectônicos, 7, Anais, p. 28-30

Amorim J.L., Rizzoto G.J., Scandolara J.E. 1999b. Terreno Jamari, região central de Rondônia: evidências de processos tafrogenéticos que precedem a convergência do início do mesoproterozóico no seguimento sudeste do Cráton Amazônico. In: SBG (NO), Sim. Geol. Amazônia, 7, Bol. Res. Exp., p. 270-273.

Braun I., Montel J-M., Nicollet C. 1998. Electron microprobe dating of monazites from high-grade gneisses and pegmatites of the Kerala Khondalite Belt, southern India. Chem.Geol., 146:65-85.

Catlos E.J., Gilley L.D., Harrison T.M. 2002. Interpretation of monazite ages obtained via in situ analysis. Chem. Geol, 188:193-215.

Cocherie A. \& Albarede F. 2001. An improved U-Th-Pb age calculation for eletron microprobe dating of monazite. Geoch. Cosmoch. Acta, 65:4509-4522.

Cocherie A., Legendre, O., Peucat J.J., Kouamelan A.N. 1998. Geochronology of polygenetic monazites constrained by in situ electron microprobe Th-U-total lead determination: implications for lead behaviour in monazite. Geoch. Cosmoch. Acta, 62:2475-2497.

Faure G. 1986. Principles of Isotopic Geology. 2nd., John Wiley \& Sons, New York. $589 \mathrm{pp}$

Foster G., Gibson H.D., Parrish R., Horstwood M., Fraser J., Tindle A. 2002. Textural, chemical and isotope insights into the nature and behaviour of metamorphic monazite. Chem. Geol., 191:183-207.

Isotta C.A.L., Carneiro J.M., Kato H.T., Barros R.J.L. 1978. Projeto Provincia Estanifera de Rondônia. Relatório Final. MME/DNPM/ CPRM, Porto Velho, vol. I, 407p.

Krogh T.E. 1973. A low-contamination method for hydrotermal decomposition of zircon and extraction of $\mathrm{U}$ and $\mathrm{Pb}$ for isotopic age determinations. Geoch. Cos 485-494.

Ludwig K.R. 1993. PBDAT. A computer program for processing Pb-UTh isotope data. USGS, Open File Report, 88:542, 34p.

Ludwig K.R. 2001. Users Mamual for Isoplot/Ex version 2.47. A geochronological toolkit for Microsoft Excel. Berkeley Geochronology Center, Sp. Public., 1a, 55p.

Montel J-M., Foret S., Veschambre M., Nicollet C., Provost A. 1996. Electron microprobe dating of monazite. Chem. Geol., 131:37-53.

Payolla B.L., Bettencourt J.S., Kozuch M., Leite Jr. W.B., Fetter A.H., Van Schmus, R. 2002. Geological evolution of the basement rocks in the central-eastern part of the Rondônia Tin Province, SW Amazonian Craton, Brazil: U-Pb and Sm-Nd isotopic constraints. Precamb. Res., 119:141-169.

Scandolara J.E., Rizzotto G.J., Silva C.R., Bahia R.B.C., Quadros M.L.E.S. 1996. Revisão da geologia do Estado de Rondônia e áreas adjacentes. In: SBG, Congr. Brás. Geol., 39. Anais, 1:47-50.

Scandolara J.E., RizzottoG.J., Amorim J.L., Quadros M.L.E.S., Bahia R.B.C. 2001. Evolução geológica do segmento sudoeste do Cráton Amazônico - Estado de Rondônia e adjacências. In: N.J. Reis \& M.A.S. Monteiro (Coord.). Contribuições à Geologia da Amazônia, 2, SBG-NO, p.251-340.

Silva L.F.S., Costi H.T., Teixeira J.T. 1995. Faciologic mapping and preliminary petrography of Palanqueta albite granite - Bom Futuro, Rondônia State (Brazil). In: IGCP-Project 315, Symp. Rapakivi Granites and Related Rocks, Abstracts Volume, Belém, p.73-74

Silva L.F.S., Costi H.T., Dall'Agnol R., Teixeira J.T. 1997. Petrographic and geochemical characteristics of the tin-bearing albite granite of Morro da Palanqueta, Bom Futuro Mine, Rondônia, Brazil. In: Intern. Symp. on Granites and Assoc. Mineraliz., 2, Salvador, p.155-
156.

Silva L.C., Armostrong R., Pimentel M.M., Scandolara J., Ramgrab G., Windler W., Angelim L.A., Vasconcelos A.M., Rizzoto G., Quadros M.L., Sander A, Rosa A L.Z. 2002. Reavalização da evolução geológica em terrenos pré-cambrianos brasileiros com base em novos dados U-Pb, SHRIMP, parte III. Províncias Borborema, Mantiqueira meridional e Rio Negro-Juruena. Rev. Brás. Geoc., 32:529-544.

Souza V.S. 2003. Evolução magmática e modelo metalogenético do sistema vulcano-plutônico estanífero Bom Futuro (RO). Tese de Doutoramento, Instituto de Geociências, Universidade de Brasilia, 240 p.

Souza V.S. \& Botelho N.F. 2002. Geologia do depósito de estanho do Bom Futuro (Rondônia) e composição dos fluidos nos sistemas de veios e greisens. In: E.L. Klein, M.L. Vasquez, L.T. Rosa-Costa (Eds.). Contribuições à geologia da Amazônia, 3, SBG-NO, p.199. 214.

Souza V.S., Botelho N.F., Dantas E.L., Gioia S.M.C. 2005. Geoquímica e isotópos de $\mathrm{Nd}$ das rochas do Complexo Jamari na área do depósito de estanho do Bom Futuro (RO). In: V.S. Souza \& A.M.C. Horbe (Coord.). Contribuições à Geologia da Amazônia, 4, SBG-NO, p. (em editoração).

Suzuki K. \& Adachi M. 1991. Precambrian provenance and Silurian metamorphism of the Tsubonosawa paragneiss in the South Kitakami terrane, Northeast Japan, revealed by the chemical Th-U total $\mathrm{Pb}$ isochron ages of monazite, zircon and xenotime. Geoch. Jour., 25:357-376.

Suzuki K. \& Adachi M. 1994. Middle Precambrian detrital monazite and zircons from the Hida Gneiss on Oki - Dogo Island, Japan: their origin and implications for the correlation of basement gneiss of Southwest Japan and Korea. Tectonophysics, 235:277-292.

Suzuki K., Adachi M., Tanaka T. 1991. Middle Precambrian provenanace of Jurassic sandstone in the Mino Terrane, central Japan: Th$\mathrm{U}$-total $\mathrm{Pb}$ evidence from an electron microprobe monazite study. Sedim. Geol., 75:141-147.

Tassinari C.C.G., Bettencourt J.S., Geraldes M.C., Macambira M.J.B., Lafon J.M. 2000. The Amazonian Craton. In: U.G. Cordani, E.J. Milani, A. Thomaz Filho, D.A. Campos (Eds.) Tectonic Evolution of South America, p. 41-95.

Tickyj H, Hartmann L.A., Vasconcellos M.A.Z. 2001. EPMA dating of monazites from southern Brazilian Shield. In: Simp. Sudamer. Geol. Isotópica, 3, Atas. CD-ROM. Pucon, Soc. Geol. Chile, v.1, p. 71-74.

Villanova M.T. \& Franke N.D. 1985. Serra do Bom Futuro-Rondônia: a volcanic-breccia pipe-hosted tin mineralization. In: Symp. Rapakivi Granites and Related Rocks, Abstracts Volume, Belém, p.83-84.

Vlach S.R.F. \& Gualda G.A.R. 2000. Microprobe monazite dating and the ages of some granitic and metamophic rocks from southeastern Brazil. Rev. Bras. Geoc., 30:214-217.

Vlach S.R.F. \& Correia C.T. 2001. A comparison between chemical and isotopic microbeam dating methods: Th-U-total $\mathrm{Pb}$ (monazite, microprobe) and U-Pb (zircon, SHIRIMP). In: Simp. Sudamer. Geol. Isotopica, 3, Atas, CD-ROM. Pucon, Soc. Geol. Chile, v.1, p.7982.

Vlach S.R.F. \& Del Lama E. 2002. Idades Th-U-Pb de monazita e geotermobarometria de rochas metapelíticas de alto grau do sistema orogênico Itabuna-Salvador-Curaça, BA, Brasil: um estudo em microssonda eletrônica. Rev. Inst. Geoc. - USP, 2:9-22.

Manuscrito NB-0063 Revisão aceita em 08 de março de 2007 